\title{
Colon en arañazo de gato. Una entidad poco conocida
}

\author{
J. S. Baudet, X. Arguiñarena, D. Díaz-Bethencourt, M. Soler y J. Avilés \\ Servicio de Aparato Digestivo. Hospital Universitario Ntra. Sra. de Candelaria. Santa Cruz de Tenerife
}

\section{CASOS CLÍNICOS}

Presentamos dos pacientes intervenidos de CCR y portadores de ileostomías. Uno, de 58 años, intervenido hace 8 meses, pendiente de reintervención para cierre de la ileostomía y otro, de 71 años, intervenido hace 5 años, que no ha querido reintervenirse para cierre de la ileostomía y reconstrucción del paso del colon.

Los pacientes acudían para colonoscopia de control. En ambos caso el colon presentaba una colitis leve con mucosa eritematosa y congestiva, con pérdida del patrón vascular, compatible con una colitis por diversión. Al insuflar aire se observó la aparición de roturas lineales de la mucosa; superficiales en el primer caso (Fig. 1); y más profundas, con visualización de la muscular de la mucosa, en el segundo (Fig. 2). En ambos casos se pudo completar la colonoscopia y los pacientes no presentaron ninguna complicación secundaria. Las biopsias se informaron como compatibles con una colitis por diversión.

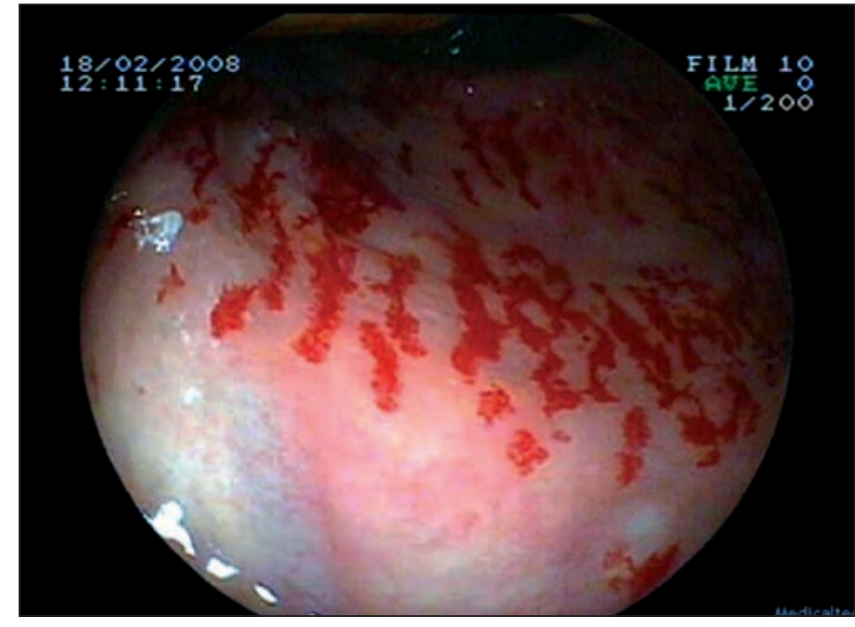

Fig. 1.

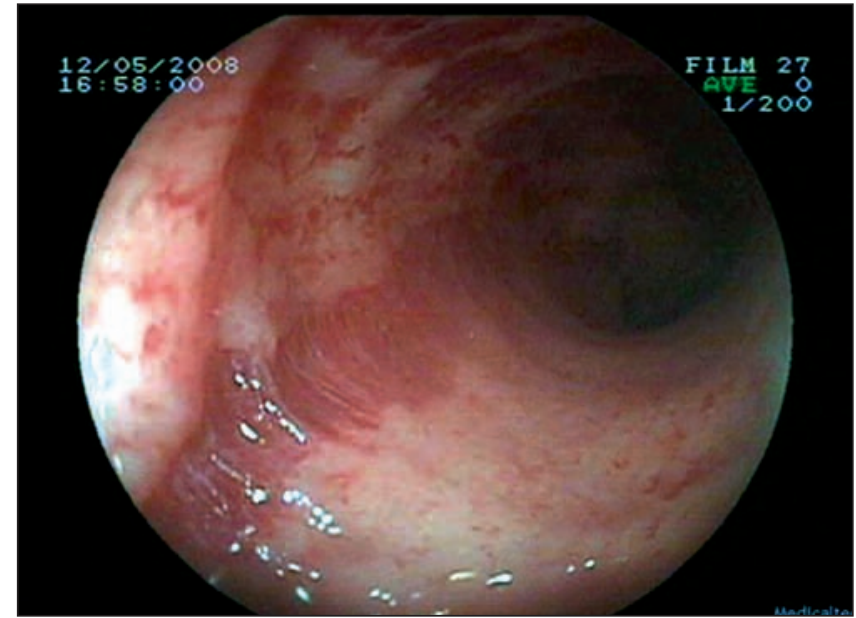

Fig. 2.

\section{COMENTARIOS}

Recientemente se ha descrito una entidad clínica que se ha dado en denominar, "cat scratch colon" (1). Se trata de la aparición durante la colonoscopia de roturas lineales de la mucosa, de color rojo brillante. Estos defectos de continuidad de la mucosa pueden ser superficiales, apareciendo como lesiones lineales que por su aspecto recuerdan al arañazo de un gato (Fig. 1) o más profundos, llegando a mostrar la muscular de la mucosa (Fig. 2). McDonnell y cols. (1), autores de esta denominación, encontraron este tipo de lesiones con una prevalencia del 0,25\% (21 pacientes) en su serie de 8.277 colonoscopias. Ellos encontraron una colitis colágena en el 14\% (3 pacientes) y mucosa normal en el $86 \%$ restante (18 pacientes). Previamente otros autores habían descrito la aparición de estas roturas de la mucosa durante la colonoscopias tanto en pacientes con colitis colágena $(2,3)$ como con colitis por diversión (4). Se trata de un hallazgo que generalmente 
no tiene repercusiones clínicas, aunque se han descrito casos de neumoperitoneo secundario al "cat scratch colon” (4).

El "cat scratch colon" se atribuye al barotrauma producido por la insuflación de aire en un colon cuya mucosa, por cualquier circunstancia (en nuestro caso una colitis por diversión, en otros una colitis colágena, en otros por alguna causa indeterminada), tiene alterada su elasticidad, como pudimos demostrar en un caso previo (5).

Nosotros hemos encontrado con relativa frecuencia este tipo de lesiones en pacientes afectos de colitis por diversión y en ninguno caso hemos observado complicaciones secundarias. Es posible que las colitis por diversión de larga evolución, como el segundo caso que presentamos (Fig. 2), estén más predispuestos a este tipo de lesiones.

\section{BIBLIOGRAFÍA}

1. McDonnell WM, Loura F, Pointon MJ, Greenson JK. Cat scratch colon. Endoscopy 2007; 39: 459-61.

2. Cruz-Correa M, Milligan F, Giardiello FM, Bayless TM, Torbenson M, Yardley JH, et al. Collagenous colitis with mucosal tears on endoscopic insufflation: a unique presentation. GUT 2002; 51: 600.

3. Mitchell JD, Teague R, Bolton R, Lowes J. Submucosal dissection in collagenous colitis. GUT 2004; 53: 470.

4. Komuro Y, Watanabe T, Hata K, Nagawa H. Diversion colitis with a mucosal tear on endoscopic insuflation. GUT 2003; 52: 1388-9.

5. Baudet JS, Diaz-Bethencourt D, Arguiñarena X, Soler M, Morales S, Avilés J. Cat scratch colon is caused by barotrauma secondary to insufflation during colonoscopy. Endoscopy 2008; 40(10): 878. 